\title{
Fatores de risco de queimaduras domésticas: conhecimento de profissionais de Unidades Estratégia Saúde da Família
}

\author{
Risk factors of domestic burns: knowledge of professionals of Strategy Family Health
}

Lucília de Cássia Freire de Oliveira†, Maria Cristina Almeida de Souza**

Como citar esse artigo. de

Oliveira, L.C.F.; de Souza, M.C.A

Fatores de risco de queimaduras

domésticas: conhecimento de

profissionais de Unidades Estratégia

Saúde da Família. Revista de

Saúde. 2019 Jan./Jun.; 10 (1): 09-14

\begin{abstract}
Resumo
Introdução: a significativa prevalência de queimadura ressalta a importância do compartilhamento de informações sobre as principais medidas de prevenção e fatores de risco associados a esse agravo. Neste contexto, destaca-se o papel dos profissionais da equipe Estratégia Saúde da Família (eSF), cujo processo de trabalho inclui o desenvolvimento de ações relacionadas à prevenção de agravos, entre os quais se incluem as queimaduras. Objetivo: avaliar o conhecimento de profissionais das Unidades ESF do Município de Vassouras/RJ sobre os fatores de risco de queimaduras domésticas. Metodologia: estudo quantitativo, transversal, cujos dados foram coletados por meio da aplicação de questionário estruturado. Resultados: atenderam ao critério de inclusão e participaram da pesquisa $74,4 \%$ dos profissionais. Sobre o local onde o ferro de passar roupas é guardado após o uso, 78,4\% dos profissionais informaram que isto se dá em local fechado, de difícil acesso às crianças; $44,1 \%$ guarda o botijão de gás dentro de casa; $15,6 \%$ dos participantes deixam cabos das panelas ficam para o lado de fora do fogão ao cozinhar. Sobre a altura das tomadas, $22,5 \%$ declararam que ficam em locais alto. O uso de extensões (pinos T’s) nas residências foi confirmado por 14,7\%. Conclusão: há necessidade da realização de atividades de educação em saúde sobre fatores de risco e medidas de prevenção de queimaduras voltadas ao profissionais que atuam nas equipes das Unidades Estratégia de Saúde do município de Vassouras/RJ, a fim de que possam ser multiplicadores dessas informações à população adscrita à unidade de saúde.
\end{abstract}

Palavras-chave: Queimaduras, Prevenção de acidentes, Educação em saúde, Emergências.

\begin{abstract}
Introduction: the significant prevalence of burns underscores the importance of sharing information about the main prevention measures and risk factors associated with this disease. In this context, the role of professionals in the Family Health Strategy (eSF) team is highlighted, whose work process includes the development of actions related to the prevention of injuries, including burns. Objective: to evaluate the knowledge of professionals of the ESF Units of the Municipality of Vassouras / RJ on the risk factors of domestic burns. Methodology: quantitative, transversal study, whose data were collected through the application of a structured questionnaire. Results: met the inclusion criteria and $74.4 \%$ of the professionals participated in the study. Regarding the place where the iron is stored after use, $78.4 \%$ of the professionals reported that this happens in a closed place, difficult to reach the children; $44.1 \%$ keep the gas cylinder in the house; $15.6 \%$ of participants leave pot ropes stand outside the stove when cooking. About the height of the outlets, $22.5 \%$ stated that they are in high places. The use of extensions (T-pins) in the residences was confirmed by $14.7 \%$. Conclusion: there is a need to carry out health education activities on risk factors and burn prevention measures aimed at the professionals who work in the teams of the Health Strategy Units of the city of Vassouras / RJ, in order to be able to multiply this information to the population attached to the health unit.
\end{abstract}

Keywords: Burns, AccidentPrevention, Health Education, Emergencies.

\section{Introdução}

Queimadura é a lesão tecidual decorrente da ação do calor, tanto direta como indiretamente. Pele, seus anexos,músculos,tendões e ossos podem ser destruídos por dano térmico. De acordo com a Organização Mundial de Saúde (OMS), saúde é um estado de completo bem-estar físico, mental e social, e não apenas a ausência de doença. No entanto, vítimas com sequelas de queimaduras não gozam de saúde por esta definiçãodada à dificuldade de tratamento ${ }^{1}$.

Queimaduras requerem, em muitos casos, tratamento hospitalar prestado por profissionais de uma equipe multiprofissional. As repercussões vão desde uma lesão superficial até a morte, dependendo da gravidade, extensão, profundidade e localização das lesões.Há possibilidade de tempo prolongado de internação, de dolorosa terapêutica e de alto custo com o tratamento de sequelas psíquicas, físicas e sociais, cujos resultados nem sempre são satisfatórios² ${ }^{2}$.

As queimaduras quase sempre resultam em transtornos psicológicos para a vítima e familiares, que necessitamde suporte psicossocial em um grande número de casos $^{3}$. Segundo o Ministério da Saúde cerca de um milhão de pessoas sofre queimaduras no Brasil

Afiliação dos autores: Mestrado em Ciências Aplicadas em Saúde da Universidade de Vassouras, Vassouras/RJ.

*Email de correpondência: mcas.souza@uol.com.br 
anualmente, com maior prevalência entre indivíduos que têmmenor condição socioeconômica ${ }^{4}$.

Noventa e cinco por cento das queimaduras ocorrem em países com baixa renda per capitacausando sofrimento, incapacidade funcional e psíquica às vítimas e até mesmo morte. Podem ocorrer também sequelas que poderiam ser evitadas por meio do conhecimento das pessoas sobre os principais fatores de risco e de medidas para prevenção ${ }^{5}$.

Comprovada que a ocorrência de queimadura é um acometimento de significativa prevalência, tornase imprescindível o compartilhamento de informações sobre seus fatores de risco e medidas de prevenção, uma importante estratégia para diminuir o número de sequelas, mortes e acidentes relacionados a esse agravo. Neste contexto, destaca-se o papel dos profissionais da equipe de Saúde da Família (eSF), que atuam no nível primário da Rede de Atenção à Saúde (RAS), consideradocomo aa "porta de entrada" do usuário aos serviços públicos de saúde.O processo de trabalho destas equipes pressupõe vínculo com os usuários e inclui o desenvolvimento de ações relacionadas à prevenção de agravos e doenças, sem prejuízo às assistenciais e curativas ${ }^{6}$.Compete também a estes profissionais, a operacionalização das atividades do Programa Saúde na Escola (PSE), Programa Interministerial, cujo escopo é a atenção à saúde de escolares, com destaque para aquelas promotoras do autocuidado, representadas por ações de educação em saúde ${ }^{7}$. O Sistema Único de Saúde (SUS), no período entre 2013 e 2014 registrou mais de 15 mil casos de internações por queimadura em crianças com idade entre 0 e 10 anos $^{8}$.

O município deVassouras está localizado na região Centro Sul do Estado do Rio de Janeiroepossui, segundo dados do Instituto Brasileiro de Geografia e Estatística (IBGE), 34.410 mil habitantes ${ }^{9}$. A cobertura populacional da Estratégia Saúde da Família é de 100\%.O município dispõe de 14 Unidades Estratégia Saúde da Família $(E S F)^{10}$.

$\mathrm{O}$ presente estudo objetivou avaliar $\mathrm{O}$ conhecimento de profissionais das Unidades ESF do Município de Vassouras/RJ sobre os fatores de risco de queimaduras domésticas.

\section{Metodologia}

Estudo quantitativo,transversal,cujos dados foram coletados no período de outubro a dezembro de 2017, por meio da aplicação de questionário estruturado contendo 15 perguntas (Figura 1). Oquestionário aplicado foi uma adaptação do utilizado por Lima Júnior et al. ${ }^{1}$ A amostra foi constituída por profissionais deequipesdas Unidades ESF do município de Vassouras/RJ.Os critérios de inclusão foram o participante ter seu nome constante na equipe da Unidade ESF disponibilizada no Cadastro
Nacional de Estabelecimentos de Saúde (CNES) e nele estar registrado de acordo com oCadastro Brasileiro de Ocupação (CBO) referente a uma das profissões que integrama composição das eSF de acordo com a Portaria MS/GM 2.436, de 21 de Setembro de 2017. Concordar em assinar o Termo de Consentimento Livre e Esclarecido (TCLE) também foi critério de inclusão. Foram excluídos os profissionais que: a) estivessem atuando em substituição a outro profissional efetivo da equipe, b) estivessem afastados da atividade profissional para tratamento de saúde, c)não concordassem em assinar o TCLE.

Essa pesquisa foi aprovada pelo Comitê de Ética em Pesquisa (CEP) da Universidade de Vassouras sob número 2.277.421, de 15/09/2017, tendo sendo respeitados todos os princípios éticos que regem a Lei 196/996 do Conselho Nacional de Saúde/Ministério da Saúde, regulamentadores da pesquisa em seres humanos e a Resolução $n^{\circ} 466$, de 12 de dezembro de $2012^{11}$.

\section{Resultados}

De um universo de 137 profissionais cadastrados nas 14Unidades ESF no CNES, atenderam ao critério de inclusão e participaram da pesquisa $102(74,4 \%)$, sendo 7 médicos, 18 enfermeiros, 3 técnicos de enfermagem, 55 Agentes Comunitários de Saúde (ACS), 8 dentistas e 13 auxiliares de saúde bucal. A idade variou de 24 a 63 anos. Houve predomínio do gênero feminino.Formação de nível superior foi declarada por $34,3 \%$ sujeitos da pesquisa.

Sobre o local onde o ferro de passar roupas é guardado após o uso, $78,4 \%$ dos profissionais informaram que isto se dá em local fechado, de difícil acesso às crianças. Sobre a localização do botijão de gás, 44,1\% dos sujeitos da pesquisa o guardam dentro de casa.

Os cabos das panelas ficam para o lado de fora do fogão ao cozinhar na casa de $15,6 \%$ dos participantes. Sobre a altura das tomadas, $22,5 \%$ declararam que ficam posicionadas em locais alto, $40,1 \%$ afirmaram que estão em locais baixo e sem uso de protetor. No entanto, $14,7 \%$ citaram que são baixas estão com uso de protetor.O uso de extensões (pinos T's) nas residências foi confirmado por $14,7 \%$ dos sujeitos da pesquisa. Sobre o manuseio de aparelhos ligados à eletricidade descalço ou molhado, $59,8 \%$ negaram. Apenas 7,8\% participantes afirmaram que nas festas de São João costumam fazer fogueiras. Não usam fogos de artifício 65,6\%de profissionais e os que usam afirmaram que os fogos são manipulados por adultos. Perguntados sobre a exposição ao sol, 83,3\% informaram que se protegem do sol por meio do uso de protetor solar e de chapéu. Os principais resultados, mostrados na tabela 1 , foram analisados por meio do cálculo de frequência e porcentagem. 


\section{I- IDENTIFICAÇÃO DA UNIDADE E DO PARTICIPANTE}

Unidade Estratégia Saúde da Família: CNES: CBO:

Participante: Gênero: ( ) F ( ) M

Idade: Profissão na equipe: Data:

\section{II- CONHECIMENTO SOBRE FATORES DE RISCO DE QUEIMADURAS DOMÉSTICAS}

1-após término de uso, o ferro elétrico fica exposto em local de fácil acesso para as crianças?

( ) $\operatorname{sim}$ ( ) não

2-onde fica localizado botijão de gás na sua casa? ( ) fora de casa ( ) dentro de casa 3-ao cozinhar, os cabos das panelas ficam para que lado do fogão? ( ) dentro ( ) fora 4-as tomadas da sua casa estão a uma altura de 1 metro do chão? ( ) sim ( ) não 5-em caso negativo, estão com protetores? ( ) sim ( ) não 6-em sua casa, são usadas extensões/pinos T's? ( ) sim ( ) não

7-manuseia aparelhos ligados à eletricidade descalço ou molhado? ( ) sim ( ) não 8-os produtos inflamáveis são guardados: ( ) junto com a comida ( ) junto com os produtos de limpeza ( ) separados em locais elevados ( ) separados em locais baixos 9-produtos ácidos soda cáustica e/ou ácido muriático são guardados em qual local?

( ) junto com comida ( ) separados, em locais elevados

( ) separados, em locais baixos ( ) não os uso

10-produtos ácidos (soda cáustica / ácido muriático) são identificados? ( ) $\operatorname{sim}$ ( )não 11-nas festas de São João, você faz fogueiras? ( ) sim ( ) não

12-as fogueiras ficam próximas de matas, produtos inflamáveis, fios elétricos?( ) sim ( ) não

13-fogos de artifícios quando são usados:

( ) são manipulados por adultos e as crianças ficam a uma distância segura

( ) são manipulados por adultos e as crianças não ficam a uma distância segura

( ) são manipulados indistintamente por crianças e adultos

( ) não os uso

14-os membros de sua família, quando se expõem ao sol:

( ) usam protetor solar / chapéu ( ) não se protegem

15-quando você vai para a praia costuma ficar exposto ao sol entre as 10 e 16 horas sem nenhuma proteção: ( ) sim ( ) não 


\begin{tabular}{|c|c|c|}
\hline Dados & $\begin{array}{c}\text { Frequência } \\
\text { absoluta } \\
\text { (n) }\end{array}$ & $\begin{array}{c}\text { Frequência } \\
\text { relativa } \\
(\%)\end{array}$ \\
\hline $\begin{array}{l}\text { Após término de uso, ferro elétrico fica exposto em local de fácil acesso } \\
\text { para as crianças }\end{array}$ & 22 & 21,5 \\
\hline Botijão de gás guardado dentro de casa & 45 & 44,1 \\
\hline Cabos de panelas voltadas para o lado de fora do fogão & 16 & 15,6 \\
\hline Tomadas, na casa, instaladas a uma distância mínima de 1 metro do chão & 23 & 22,5 \\
\hline $\begin{array}{l}\text { Tomadas, na casa, instaladas a uma distância inferior } 1 \text { metro do chão e } \\
\text { estão desprovidas de protetor }\end{array}$ & 41 & 40,1 \\
\hline $\begin{array}{l}\text { Tomadas, na casa, estão instaladas a uma distância inferior } 1 \text { metro do } \\
\text { chão e estão providas de protetor }\end{array}$ & 15 & 14,7 \\
\hline Manuseia eletrodomésticos quando estão com pés molhados ou descalços & 41 & 40,1 \\
\hline Produtos ácidos soda cáustica e/ou ácido muriático são guardados & \multirow[t]{3}{*}{-} & \multirow[t]{3}{*}{-} \\
\hline () junto com comida & & \\
\hline () separados, em locais elevados & & \\
\hline \multirow[t]{3}{*}{ () separados, em locais baixos } & 00 & 00 \\
\hline & 24 & 23,5 \\
\hline & 25 & 24,5 \\
\hline () não os uso & 53 & 51,9 \\
\hline Produtos inflamáveis armazenados junto com comida & 00 & 00 \\
\hline Fazem fogueiras nas festas de São João & 08 & 7,8 \\
\hline Utilizam extensão tipo pino "T" em casa & 15 & 14,7 \\
\hline Manipulam fogos de artificio & 35 & 34,3 \\
\hline $\begin{array}{l}\text { Membros da familia usam protetor solar ou chapéu quando se expõem ao } \\
\text { sol }\end{array}$ & 75 & 73,5 \\
\hline Usam protetor durante exposição solar entre as 10 e 16 horas & 85 & 83,3 \\
\hline
\end{tabular}

Tabela 1. Conhecimento dos profissionais de unidades Saúde da Família sobrefatores de risco para queimaduras domésticas

\section{Discussão}

Queimaduras são responsáveis por um alto índice de mortalidade. Apenas acidentes de transporte e homicídios possuem números superiores de mortes 12. Em alguns casos, é possível preveni-la, ratificando assim a importância de ações de educação em saúde pelos profissionais da área, cuja conhecimento precisa ser estimado e atualizado. Dentre as medidas de prevenção, foram citadas a implementação de legislação e políticas específicas, campanhas de prevenção voltadas ao público-alvo, ações educativas a serem desenvolvidas no ambiente escolar e nos principais meios de comunicação $0^{5,13,14,15}$. A melhoria da educação sobre queimaduras desencadeou uma revolução nos países de alta renda - a mortalidade por queimaduras está diminuindo ${ }^{5}$.

Em relação ao ferro de passar roupa, observouse que a maioria dos participantes $(78,4 \%)$ relatou que, ao terminar de usá-lo, não o deixava exposto em locais de fácil acesso às crianças, resultado semelhante ao registrado $(71,2 \%)$ por Lima Júnior et al. ${ }^{1}$.Constatou-se que guardam botijão de gás dentro de casa, $44,1 \%$ dos profissionais das Unidades ESF. Ainda que seja uma prevalência inferior à informada em outras pesquisas 1, alerta para a importância de ações de educação no sentido de que o armazenamento seja feito por todos em área externa da residência.

Ao cozinhar, 84,3\%dos entrevistados informaram que colocavam os cabos de suas panelas para dentro do fogão, corroborando os achados de outras pesquisas, que registram $66,3 \%$ adotam essa atitude correta ${ }^{1}$. Esse resultado vai ao encontro de investigação científica sinalizadora de que, em sua maioria, os fatores de risco estão relacionados à cozinha (fogão, panela e alimentos quentes, fósforo e isqueiro, fogo) e durante o preparo das refeições, principalmente com panelas e fogão $3,13,14$. Adicionalmente, significativa prevalência $(32,5 \%)$ de lesões causadas por água quente foi encontrada por Bessa et al. ${ }^{2} \mathrm{em}$ estudos revisadospor Meschial et al., $2016^{15}$.

Ainda que os resultados dessa pesquisa tenham evidenciado menor prevalência de uso de extensões ou pinos T's $(14,7 \%)$ em relação ao registrado em estudos semelhantes $(81,3 \%)^{1}$, há evidente necessidade de conscientização da população e dos profissionais das Unidades ESF do município de Vassouras para o comprometimento da segurança ao fazerem uso desses dispositivo.De acordo com 77,4\% dos entrevistados, as tomadas em suas residências situavam-se a uma distância adequada do chão, superior aos resultados de Lima Júnior et al. ' , cuja pesquisa encontrou $60 \%$ 
dos participantes com relato dessa condição. Estudo realizado por Takejima et al. (2011) revelou que somente $16,36 \%$ dos participantes possuíam algum tipo de material de prevenção domiciliar, como protetores de tomada ${ }^{14}$. Existência de tomadas localizadas a uma distância inferior a 1 metro do chão, ainda que providas de protetor, foi relatada por $14,7 \%$ dos profissionais das Unidades ESF, superando os achados de outras pesquisas, representando um alerta sobre o compartilhamento de informações principalmente aos que possuem crianças em casa.

Já em relação ao manuseio da eletricidade com pés descalço e/ou molhado, evidenciou-se que significativa parcela dos profissionais não o realizava $(40,1 \%)$, situação também observada por Lima Junior et al. ${ }^{1}$ em $66,3 \%$ da amostra estudada.

Os produtos inflamáveis localizavam-se, principalmente, em locais separados e elevados (50\%), e apenas $15 \%$ não utilizavam esses produtos, sendo que, do total que os utilizavam, $81 \%$ identificavam-nos de forma correta. Os produtos ácidos apresentaram uma predominância da não utilização, representando $51,9 \%$ das respostas, valor muito próximo ao encontrado por outros pesquisadores ${ }^{1}$.

Na época do São João apenas 7,8\% relataram fazer fogueiras, muito provavelmente por não representar tradição arraigada na região, diferentemente dos resultados encontrados em estudo realizada no Ceará(que registrou $71,3 \%$ de informação positiva) ${ }^{1}$ e também na pesquisa de Fernandes et al. ${ }^{16}$, que identificou uma incidência maior de queimaduras nos meses de junho devido aos festejos juninos. Assim, observou-se que a maioria dos entrevistados $(65,7 \%)$ não fazia uso de fogos de artifício, aproximando-se ao relatado por Lima Júnior et al. ${ }^{1}(58,8 \%)$. Bessa et al. ${ }^{2}$ ratificam que a fabricação, venda e uso de fogos de artifícios, assim como produtos e líquidos inflamáveis para crianças, sem leis adequadas e fiscalização que os coíbam, levam a verdadeiros dramas causados pela imprudência e ignorância. Em relação à exposição solar, notou-se que $73,5 \%$ faziam uso de protetor solar e/ou chapéu, quando se expunham ao sol.

\section{Conclusão}

Esta pesquisa permitiuverificarque hánecessidade de promoção de atividades de educação em saúde sobre o tema queimaduras para os profissionais das Unidades ESF do município de Vassouras/RJ. Não são todos os profissionais que adotam, em seu cotidiano, as medidas de prevenção de queimaduras. É imprescindível que, como formadores de opinião e possíveis multiplicadores de informação e profissionais que atuam na assistência, estejam atualizados sobre os principais fatores de risco e medidas de prevenção sobre o agravo.

\section{Referências}

1. Lima Júnior EM, Melo MCA, Alves CC, Alves EP, Parente EA, Ferreira GE. Avaliação do conhecimento e promoção da conscientização acerca da prevenção de queimaduras na população de Fortaleza-CE. RevBras Queimaduras 2014;13(3):161-7. Disponível em <http://www.rbqueimaduras. com.br/details/216/pt-BR/avaliacao-do-conhecimento-e-promocao-daconscientizacao-acerca-da-prevencao-de-queimaduras-na-populacao-defortaleza---ce> Acesso em 2 de Julho de 2018.

2. Bessa DF, Ribeiro ALS, Barros SEB, Mendonça MC, Bessa IF, Alves MA et al. Perfil Epidemiológico dos Pacientes Queimados no Hospital Regional de Urgência e Emergência de Campina Grande - Paraíba - Brasil.RevBrasCi Saúde 2006;10(1):73-80. Disponível em <http://www.periodicos.ufpb.br/ ojs/index.php/rbcs/article/view/3432/2809> Acesso em 2 de Dezembro de 2018.

3. Gimeniz-Paschoal SR, Pereira DM, Nascimento EM. Efeito de ação educativa sobre o conhecimento de familiares a respeito de queimaduras infantis em ambientes domésticos. Rev Latino-am Enfermagem 2009 maiojunho; 17(3). Disponível em <http://www.scielo.br/pdf/rlae/v17n3/pt_10. pdf $>$ Acesso em 23 de Maio de 2018.

4. Brasil. Governo do Brasil. Primeiros socorros. Publicado em 23/12/2017. Disponível em <http://www.brasil.gov.br/noticias/saude/2017/06/ummilhao-de-brasileiros-sofrem-queimaduras-por-ano> Acesso em 27 de Agosto de 2018.

5. Tevlin R, Dillon L, Clover AJP. Education in burns: lessons from the past and objectives for the future. Burns. 2017 Sep;43(6):1141-1148. Disponível em <https://www.ncbi.nlm.nih.gov/pubmed/28408146> Acesso em 15 de Junho de 2018

6. Brasil. Ministério da Saúde. Portaria nº. 2.436, de 21/09/2017. Aprova a Política Nacional de Atenção Básica, estabelecendo a revisão de diretrizes para a organização da Atenção Básica, no âmbito do Sistema Único de Saúde (SUS) . Disponível em <http://www.saude.df.gov.br/wp-conteudo/uploads/2018/04/ Portaria-n\%C2\%BA-2436-2017-Minist $\%$ C3\%A9rio-da-Sa $\%$ C3\%BAdeAprova-a-Pol $\%$ C3 $\%$ ADtica-Nacional-de-Aten $\% \mathrm{C} 3 \% \mathrm{~A} 7 \% \mathrm{C} 3 \% \mathrm{~A} 3 \mathrm{o}$ B $\%$ C3\%A1sica..pdf $>$ Acesso em 21 de Agosto de 2018 .

7. Brasil. Presidência da República. Decreto nº 6286, de 5 de Dezembro de 2007. Institui o Programa Saúde na Escola - PSE e dá outras providências. Disponível em <http://www.planalto.gov.br/ccivil 03/ Ato2007-2010/2007/ Decreto/D6286.htm> Acesso em 28 de Maio de 2018 .

8. Brasil. Ministério da Saúde. Secretaria de Atenção à Saúde. Departamento de Atenção Especializada. Cartilha para tratamento de emergência das queimaduras / Ministério da Saúde, Secretaria de Atenção à Saúde, Departamento de Atenção Especializada. - Brasília: Editora do Ministério da Saúde, 2012. Disponível em <http://bvsms.saude.gov.br/bvs/public acoes/cartilha tratamento emergencia queimaduras.pdf $>$ Acesso em 10 de Dezembro de 2018 .

9-. Brasil. Instituto Brasileiro de Geografia e Estatística (IBGE). Dados populacionais. Disponível em $<$ https://cidades.ibge.gov.br/brasil/rj/ vassouras/panorama> Acesso em 15 de Junho de 2018.

10. Brasil. Ministério da Saúde. Informação e Gestão da Atenção Básica. Cobertura da Atenção Básica. Disponível em <http://datasus.saude.gov. br/sistemas-e-aplicativos/epidemiologicos/siab> Acesso em 25 de Julho de 2018 .

11. Brasil. Ministério da Saúde. Conselho Nacional de Saúde. Resolução $n^{\circ} 466$, de 12 de dezembro de 2012. Diretrizes e normas regulamentadoras de pesquisa envolvendo seres humanos. Brasília: Diário Oficial da União. República Federativa do Brasil. 2013 jun 13;150(112 Seção 1):59-62. Disponível em <http://conselho.saude.gov.br/resolucoes/2012/reso466.pdf> Acesso em 2 de Março de 2019.

12. Pan R, Silva MTR, Fidelis TLN, Vilela LS, Silveira-Monteiro CA, Nascimento LC. Conhecimento de profissionais de saúde acerca do atendimento inicial intra-hospitalar ao paciente vítima de queimaduras. Rev Gaúcha Enferm. 2018;39:e2017- 0279. Disponível em <

http://www.scielo.br/pdf/rgenf/v39/1983-1447-rgenf-39-e2017-0279.pdf> Acesso em 2 de Março de 2019.

13. Lemos EH, Ribeiro ER. Prevenção de queimaduras na infância. Caderno Saúde e Desenvolvimento 2013; 2(2):45-52.

14. Takejima ML, Netto RFB, Toebe BL, Andretta MA, Prestes MA, Takak 
JL. Prevenção de queimaduras: avaliação do conhecimento sobre prevenção de queimaduras em usuários das unidades de saúde de Curitiba. RevBras Queimaduras. 2011;10(3):85-8. Disponível em $<$ http://www.rbqueimaduras. com.br/details/74/pt-BR/prevencao-de-queimaduras--avaliacao-doconhecimento-sobre-prevencao-de-queimaduras-em-usuarios-das-unidadesde-saude-de-curitiba> Acesso em 2 de Março de 2019.

15. Meschial WC, Sales CCF, Oliveira MLF. Fatores de risco e medidas de prevenção das queimaduras infantis: revisão integrativa da literatura. RevBras Queimaduras. 2016;15(4):267-73. Disponível em <http://www. rbqueimaduras.com.br/details/325/pt-BR/fatores-de-risco-e-medidas-deprevencao-das-queimaduras-infantis--revisao-integrativa-da-literatura $>$ Acesso em 3 de Março de 2019.

16. Biscegli TS. Profile ofchildrenandadolescentsadmittedto a BurnCare Unit in thecountrysideofthestateof São Paulo. Rev Paul Pediatr. 2014; 32(3):

177-82. Disponível em <https://www.sciencedirect.com/science/article/pii/ S2359348215300075> 